AperTO - Archivio Istituzionale Open Access dell'Università di Torino

\title{
Idiopathic macular telangiectasia.
}

\section{This is the author's manuscript}

Original Citation:

\section{Availability:}

This version is available http://hdl.handle.net/2318/95237

since

Terms of use:

Open Access

Anyone can freely access the full text of works made available as "Open Access". Works made available under a Creative Commons license can be used according to the terms and conditions of said license. Use of all other works requires consent of the right holder (author or publisher) if not exempted from copyright protection by the applicable law. 


\title{
Idiopathic Macular Telangiectasia
}

\author{
Lawrence A. Yannuzzi, MD; Anne M. C. Bardal, MD; K. Bailey Freund, MD; \\ Kuan-Jen Chen, MD; Chiara M. Eandi, MD; Barbara Blodi, MD
}

Objectives: To review the frequency and nature of idiopathic macular telangiectasia and to classify the disorders based on new clinical and imaging observations.

Methods: A combined retrospective and prospective analysis of newly diagnosed patients seen over a period of 3 years. Patients were identified based on the GassBlodi classification and were studied with biomicroscopy, fluorescein angiography, and optical coherence tomography.

Results: Ten patients associated with aneurysmal telangiectasia (Gass-Blodi group 1) and 26 patients with perifoveal telangiectasia (Gass-Blodi group 2) were recruited. None with occlusive telangiectasia (Gass-Blodi group 3) were identified. New observations based on clini- cal, fluorescein angiographic, and optical coherence tomographic findings were made.

Conclusions: Our series was similar to that in the GassBlodi study in terms of frequency. New observations in groups 1 and 2 have expanded our knowledge of the clinical spectrum of these disorders. A simplified classification termed idiopathic macular telangiectasia with 2 distinct types (type I, or aneurysmal telangiectasia, and type II, or perifoveal telangiectasia) was proposed to produce a better understanding of the entities and to enhance teaching and research. The third type, occlusive telangiectasia, has been omitted from our classification based on its rarity and presence of capillary nonperfusion rather than macular telangiectasia as the primary abnormality.

Arch Ophthalmol. 2006;124:450-460
Author Affiliations: LuEsther T. Mertz Retinal Research Center, Manhattan Eye, Ear, and Throat Hospital, New York, NY (Drs Yannuzzi, Bardal, Freund, Chen, and Eandi); Department of Clinical Physiopathology, Eye Clinic, University of Torino, Torino, Italy (Dr Eandi); and Department of Ophthalmology and Visual Science, University of Wisconsin, Madison (Dr Blodi).

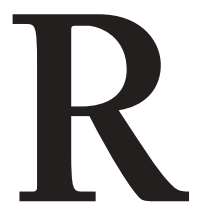

ETINAL CAPILLARY TELANGIectasia or dilated retinal capillaries are usually the result of antecedent retinal vascular inflammatory or occlusive disease. In the macula, diabetic retinopathy, hypertension, venous occlusion, inflammatory diseases, and blood dyscrasias are the usual causative factors. However, there are other forms of telangiectasia that develop in the macula and perifoveolar areas without a known cause. In 1993, Gass and Blodi ${ }^{1}$ examined 140 such cases seen at Bascom Palmer Eye Institute, Miami, Fla, over a 28-year period and established a classification of these entities with subgroups and stages. This description, termed idiopathic juxtafoveolar retinal telangiectasis, although somewhat complex, is commonly used today by most retinal specialists. In recent years, newly recognized manifestations have expanded and refined the clinical spectrum of these macular vasculopathies. Furthermore, the use of highspeed angiography and optical coherence tomography (OCT) have provided a better understanding of the nature of the vascular abnormalities and their secondary effects in the macula, to some degree paralleling histopathological observations described in the ophthalmic literature..$^{2-9}$ The purpose of this study was to describe new clinical and OCT observations in these entities and to propose a modified classification-essentially a revision and simplification of the GassBlodi model that we refer to as idiopathic macular telangiectasia (IMT) - based on these newly recognized clinical and imaging manifestations.

\section{METHODS}

Newly diagnosed patients with IMT were examined consecutively in the private offices of Vitreous-Retina-Macula Consultants of New York, New York, and at the LuEsther T. Mertz Retinal Research Center, Manhattan Eye, Ear, and Throat Hospital, New York, from January

\section{For editorial comment see page 573}

1, 2002, through December 31, 2004. The office sites included primary centers such as the hospital clinics, a secondary referral private retinal practice in Brooklyn, New York, and a mixed retinal facility that was partially a tertiary referral practice in Manhattan, New York. Only pa- 

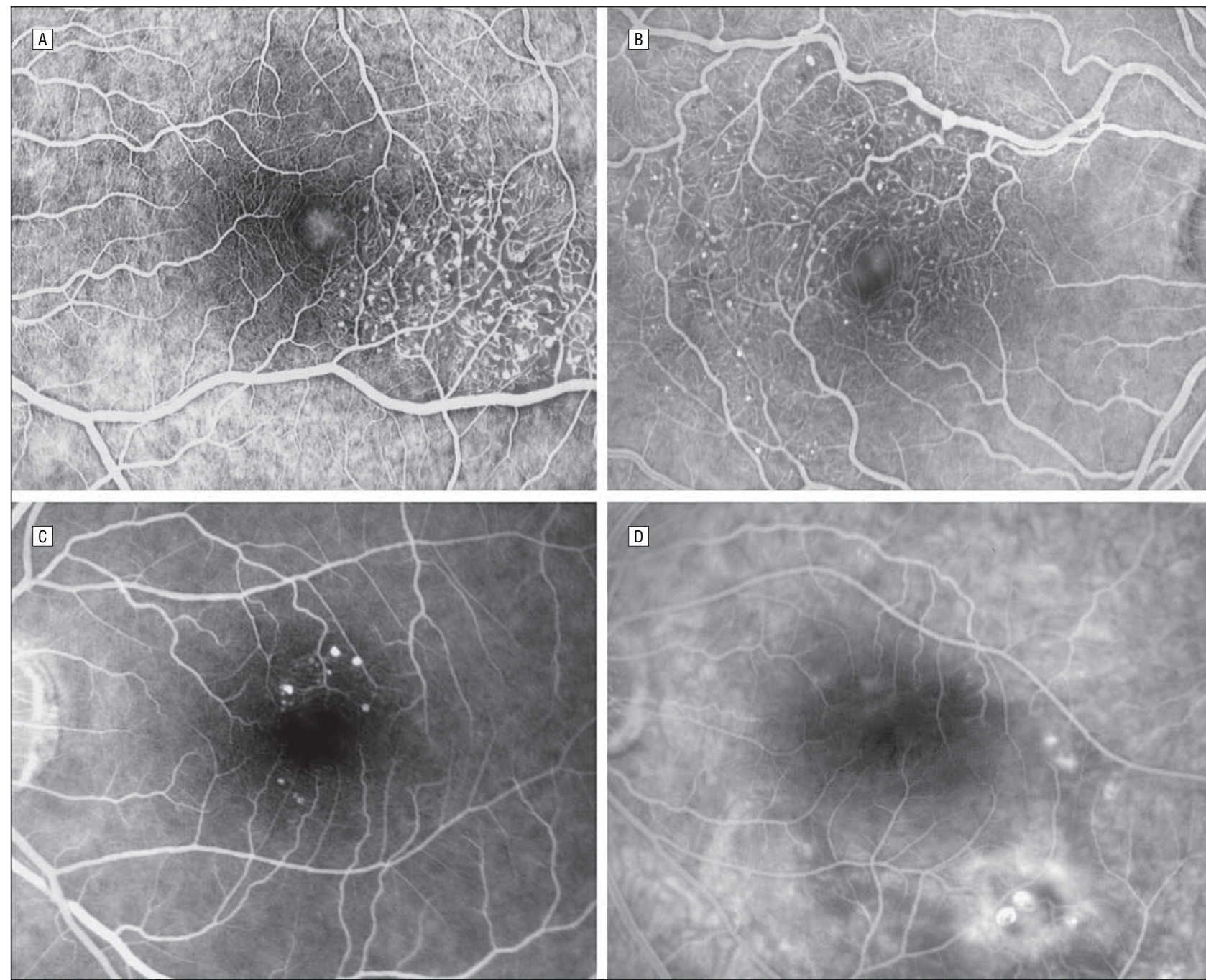

Figure 1. Aneurysmal telangiectasia with variable manifestations. The fluorescein angiograms show telangiectasia, microaneurysms, and macular edema (A), an extramacular lesion associated with minimal capillary nonperfusion (B), slightly larger aneurysms (C), and larger aneurysms with surrounding leakage (D).

tients examined for the first time who had a diagnosis made or confirmed were included. The study patients in the first 2 years were identified retrospectively; in the last year, they were recruited prospectively. A careful history on related systemic diseases, specifically systemic hypertension, diabetes mellitus, and radiation therapy to the head, was obtained from each patient. A full clinical examination, including indirect ophthalmoscopy and slitlamp biomicroscopy with the Goldmann contact lens, was performed. Digital fundus photography, digital fluorescein angiography (FA), and OCT were carried out for all of the patients.

The patients were classified into 3 groups. Type 1 is aneurysmal telangiectasia (Figure 1). This involves predominantly unilateral telangiectasia mostly in men with variably sized aneurysms. Type 2 is perifoveal telangiectasia (Figure 2 and Figure 3). This involves bilateral telangiectasia limited to the perifoveolar area without visible aneurysms and associated with subretinal neovascularization (SRN). Nonproliferative perifoveal telangiectasia refers to exudative telangiectasia and foveal atrophy (Figure 2). Proliferative perifoveal telangiectasia refers to proliferative changes with subretinal neovascularization and fibrosis (Figure 3). Type 3 is occlusive telangiectasia (Figure 4). This involves paramacular telangiectasia in an area bordering on perifoveal capillary nonperfusion.

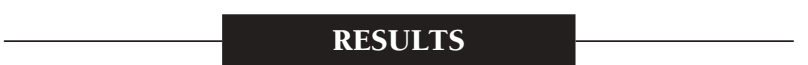

In the 3-year period of the study, a total of 36 patients with IMT were seen; 10 of the patients had aneurysmal telangiectasia, 26 had perifoveal telangiectasia, and none had occlusive telangiectasia. The case frequency ratio of these groups was 1.0:2.6:0.0 cases, respectively.

\section{TYPE 1: ANEURYSMAL TELANGIECTASIA}

The aneurysmal telangiectasia cases are described in Table 1. In this group, there were 10 patients, 7 of whom were male and 3 of whom were female. Nine patients had unilateral disease. There was only 1 bilateral case in a woman. The age range of the patients was 42 to 71 years, with a mean age of 56 years. One patient had systemic hypertension. Otherwise, there was no evidence of related systemic diseases.

The clinical manifestations varied. A consistent feature of the microangiopathy, however, was multiple capillary, venular, and arteriolar aneurysms. Aneurys- 

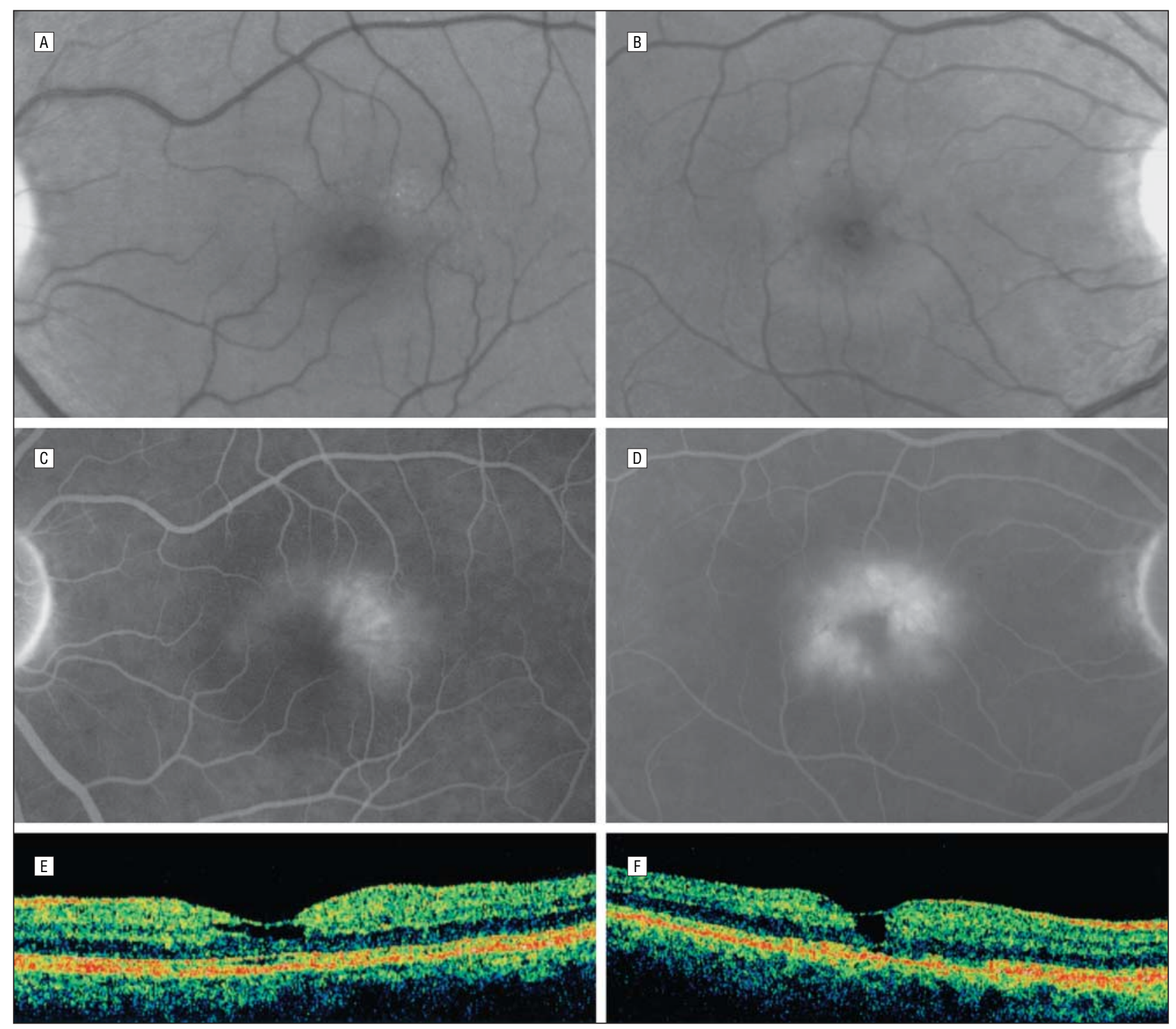

Figure 2. Perifoveal telangiectasia in the nonproliferative stage, with the reduction in retinal transparency surrounding the foveolar area on red-free photographs of the left (A) and right (B) eyes, bilateral exudative telangiectasia on the fluorescein angiograms of the left (C) and right (D) eyes, and an inner lamellar cystic change evident on optical coherence tomographic imaging in each eye, with no intraretinal cystic change within the retina or macular detachment and with retinal thickening present around fovea in the left $(\mathrm{E})$ and right $(\mathrm{F})$ eyes.

mal and telangiectatic abnormalities were evident in the superficial and deep retinal capillary circulations. Larger aneurysms were confined to the superficial circulation. In addition, some patients had minimal, patchy nonperfusion or capillary ischemia and lipid deposition. The lipid was noted particularly in association with numerous small capillary abnormalities or persistent, focal, larger aneurysms (Figure 5). There were no crystalline vitreoretinal interface deposits, plaques of pigment epithelial hyperplasia, or areas of pigment migration into the retina. In 2 patients, the pathological abnormality also varied in its location, as there were areas of involvement not only in the juxtafoveolar and extramacular regions but also in the peripheral fundus. Some larger macroaneurysms were associated with hemorrhage. The central macular vascular abnormalities were associated with edema or cystic change in the retina, seen clinically and angio- graphically and confirmed with OCT imaging in all of the cases (Figure 6). No patient had preretinal neovascularization or SRN despite the presence of capillary ischemia.

\section{TYPE 2: PERIFOVEAL TELANGIECTASIA}

The patients with perifoveal telangiectasia are described in Table 2. In this group, there were 26 patients, 11 of whom were male and 15 of whom were female. The age range was 38 to 82 years, with a mean age of 59 years. All of the patients had bilateral disease. In a few cases, there was a degree of asymmetry with regard to severity. Five patients (19.2\%) had diabetes mellitus with no clinical diabetic retinal microangiopathy. This frequency does not differ from the incidence (18.5\%) reported in the general population of the same age. ${ }^{10}$ Five patients had systemic hypertension but no corre- 

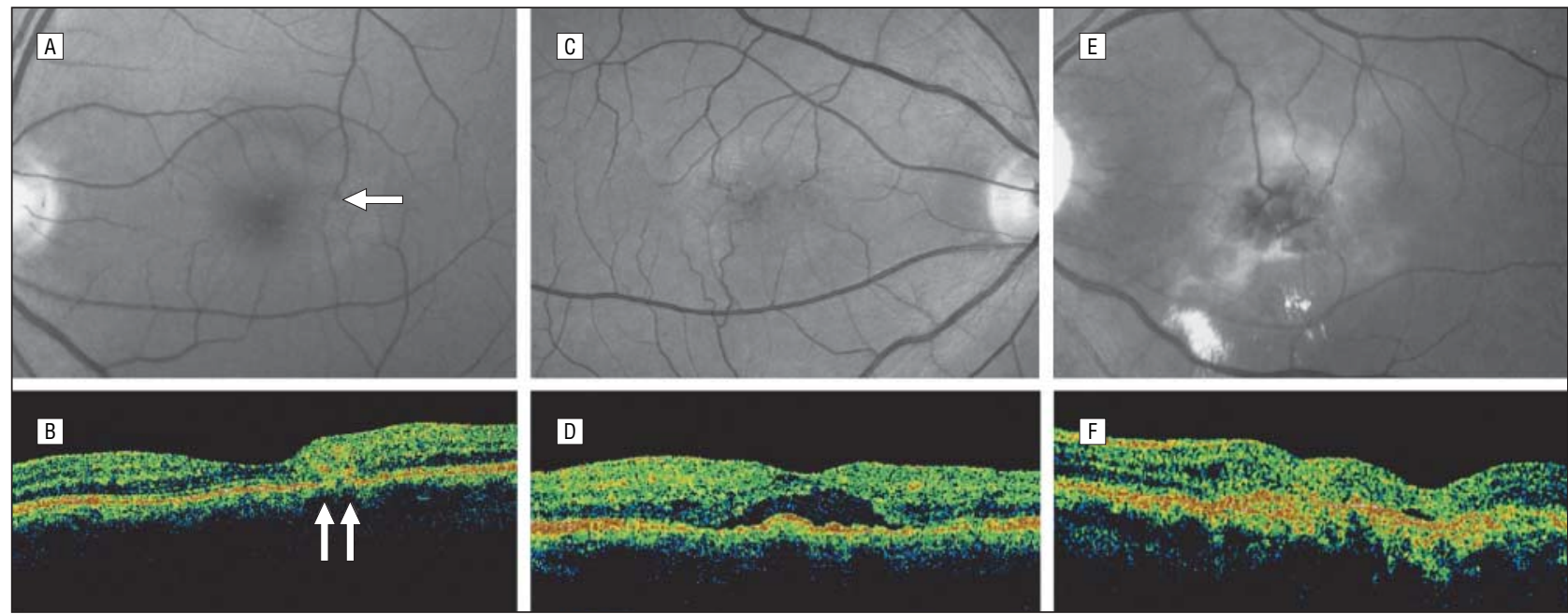

Figure 3. Perifoveal telangiectasia in the proliferative stage. Retinal vessels decussate to a subretinal plaque (arrow) with early subretinal neovascularization (A) to produce photoreflectance on optical coherence tomography (arrows) (B). More advanced subretinal neovascularization (C) is associated with macular detachment, as shown by the corresponding optical coherence tomographic image (D). Subretinal neovascularization has led to disciform scarring (E) and some cystic change in the retina, as shown by the corresponding optical coherence tomographic image (F).

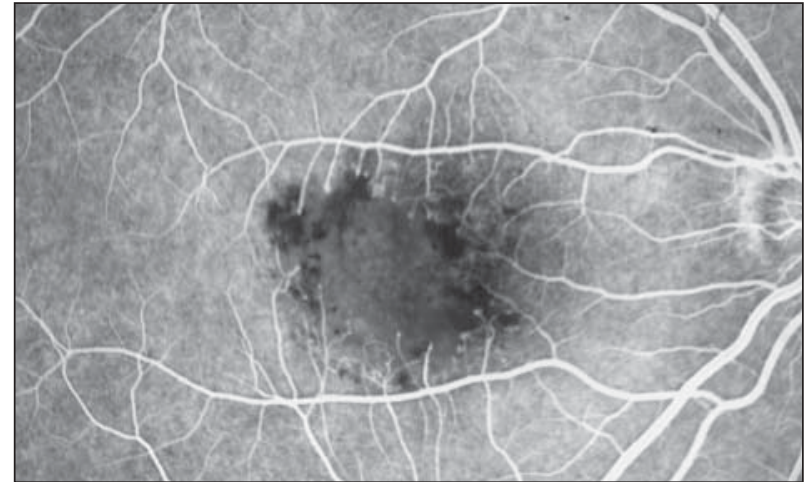

Figure 4. Occlusive telangiectasia. The fluorescein angiogram reveals ischemia within the foveolar region, bordered by a few telangiectatic vessels compensating for the capillary nonperfusion (courtesy of Richard Spaide, MD).

\begin{tabular}{|lll|}
\hline \multicolumn{3}{|c|}{ Table 1. Aneurysmal Telangiectasia } \\
$\begin{array}{ccc}\text { Patient No./ } \\
\text { Sex/Age, } \mathbf{y}\end{array}$ & $\begin{array}{c}\text { Eye(s) } \\
\text { of Lesion }\end{array}$ & \multicolumn{1}{c|}{ Systemic Disease } \\
\hline 1/M/42 & OD & NA \\
$2 / F / 71$ & OS & NA \\
3/M/53 & OD & NA \\
4/M/67 & OD & NA \\
5/M/61 & OS & Hypertension, cardiovascular disease \\
6/M/43 & OS & NA \\
$7 / F / 51$ & OU & Intracranial meningioma \\
$8 / F / 56$ & OS & NA \\
9/M/42 & OS & NA \\
10/M/59 & OS & NA \\
\hline
\end{tabular}

Abbreviation: NA, not applicable.

sponding retinal finding. Otherwise, there was no other related systemic disease in this group.

In contrast to the aneurysmal telangiectasia cases, the perifoveal telangiectasia cases were all well defined by their clinical and imaging manifestations. Early clinical changes consisted of a mild loss of transparency of

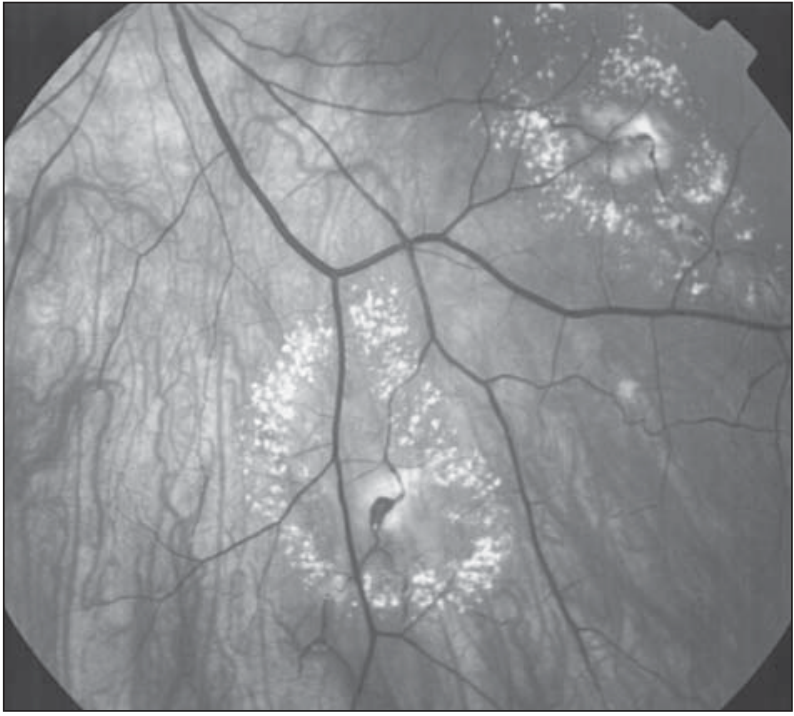

Figure 5. Aneurysmal telangiectasia. There is telangiectasia with lipid surrounding an extramacular large aneurysm.

the retina, usually in the temporal juxtafoveolar area but surrounding the fovea in more advanced cases (Figure 2A and B). This loss of transparency was typically gray and formed a partial or complete horizontal oval surrounding the fovea. In these cases, the telangiectatic vessels were absent or barely evident clinically. Retinal thickening was also difficult to judge in some eyes, but it was readily evident with OCT imaging (Figure 2E). When visible, the telangiectatic vessels were seen in the inner and outer retinal circulations. Patients with more advanced stages of the disease had a prominent dilation of capillaries.

In patients with early clinical manifestations of perifoveal telangiectasia, fluorescein angiography showed mild, diffuse intraretinal leakage or staining. In patients with more prominent telangiectasis, fluorescein leakage in the superficial circulation could be seen stereoscopically to cascade over the deep capillary leakage 

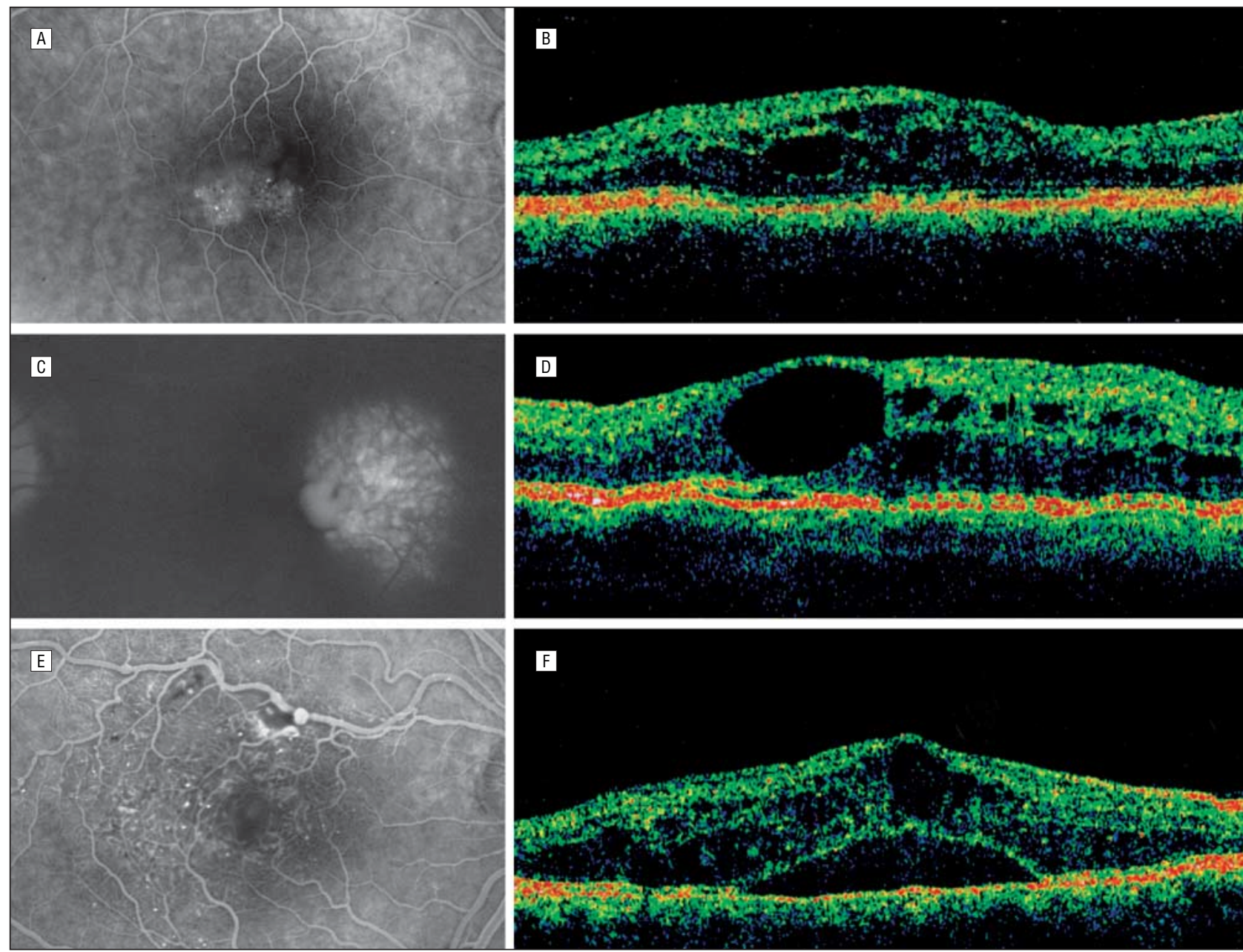

Figure 6. Aneurysmal telangiectasia. A, The fluorescein study shows telangiectatic changes in the temporal macular region associated with macular edema. B, Optical coherence tomography shows intraretinal cystic spaces and retinal thickening. C, There is leakage on the fluorescein angiogram from aneurysmal and telangiectatic changes. D, Optical coherence tomography shows a large cystic space near and within the foveola. E, Leakage from the retina has extended beneath the fovea to produce a localized detachment. F, Optical coherence tomography shows intraretinal cystic change and macular detachment.

(Figure 7). The superficial retinal circulations in 6 cases revealed segmental or sectoral dilation overlying the dilated deep retinal telangiectatic vessels. In these eyes, some capillary aneurysmal changes occurred, but there was no lipid ischemia or preretinal neovascularization. No cystic spaces were evident in the perifoveal area despite the fluorescein leakage (Figure 2). At the fovea itself, there was a circular or ovoid depression that proved with OCT imaging to be an inner lamellar cyst (Figure 2). Optical coherence tomographic imaging also showed preservation of the inner limiting membrane that constituted the anterior aspect of the cyst. More advanced cases revealed a deeper inner lamellar cyst with further loss of the outer retina and a correlating visual decline

\section{(Figure 8).}

There were no prominent aneurysms, hemorrhage, or lipid accumulation. Vitreoretinal interface crystalline deposits appeared at various stages of the disease as an inconsistent but characteristic finding. Some eyes also revealed subretinal plaques of pigmentation and dilated right-angle retinal vessels (Figure 3 ). In some cases, the dilated vessels were venular; in others, they were arteriolar in nature. A few actually had a retinal-retinal anas- tomosis (RRA) within the retina or extending to communicate with new vessels beneath the retina or SRN (Figure 9). The SRN appeared to originate from the deep retinal circulation. The development of SRN led to neurosensory detachment, subretinal hemorrhage, fibrosis, and visual decline in advanced stages of the disease (Figure 3). Cystic change in the retina, detachment of the macula, and subretinal photoreflectance from fibrovascular manifestations were evident at this stage with OCT imaging (Figure 3).

\section{TYPE 3: OCCLUSIVE TELANGIECTASIA}

Occlusive telangiectasia was not seen in this series. This is consistent with its rarity.

\section{COMMENT}

In 1982, Gass and Owakawa ${ }^{11}$ proposed a classification of idiopathic macular telangiectasia termed idiopathic juxtafoveal retinal telangiectasis. It was revised most recently in 1993 into 3 distinct groups, each with a presumed inde- 
pendent etiology, on the basis of biomicroscopical and fluorescein angiographic features. ${ }^{1}$ Subgroups based principally on demographic difference or clinical severity were also established in this classification.

For example, 39 cases were classified as group 1 (equivalent to aneurysmal telangiectasia in our series). A subgroup, group 1A, was based on the clinical detection of the aneurysmal and telangiectatic exudative vessels in the juxtafoveolar area. Group 1B (a subset of group

\begin{tabular}{|c|c|c|c|}
\hline $\begin{array}{l}\text { Patient No./ } \\
\text { Sex/Age, y }\end{array}$ & $\begin{array}{l}\text { Eye(s) } \\
\text { of Lesion }\end{array}$ & $\begin{array}{c}\text { Eye } \\
\text { With SRN }\end{array}$ & Systemic Disease \\
\hline $1 / F / 45$ & OU & NA & NA \\
\hline 2/M/65 & OU & NA & Cardiovascular disease \\
\hline $3 / F / 55$ & OU & NA & Breast cancer \\
\hline $4 / F / 51$ & OU & NA & NA \\
\hline $5 / F / 60$ & OU & NA & NA \\
\hline $6 / F / 59$ & OU & NA & Hypertension \\
\hline 7/M/55 & OU & OD & Diabetes mellitus, hypertension \\
\hline $8 / F / 59$ & OU & NA & Breast cancer \\
\hline 9/M/68 & OU & NA & NA \\
\hline $10 / F / 68$ & OU & NA & Diabetes mellitus, heart disease \\
\hline $11 / \mathrm{M} / 73$ & OU & NA & Hypertension \\
\hline $12 / F / 58$ & OU & NA & Diabetes mellitus \\
\hline 13/M/51 & OU & OS & NA \\
\hline $14 / F / 65$ & OU & NA & NA \\
\hline $15 / \mathrm{M} / 60$ & OU & OD & Diabetes mellitus \\
\hline 16/M/54 & OU & OD & NA \\
\hline $17 / F / 82$ & OU & OS & Hypertension, stroke \\
\hline $18 / F / 52$ & OU & NA & NA \\
\hline 19/M/79 & OU & NA & Hypertension, prostate cancer \\
\hline 20/F/57 & OU & OS & NA \\
\hline $21 / F / 52$ & OU & OD & NA \\
\hline 22/M/54 & OU & OD & NA \\
\hline 23/M/38 & OU & NA & NA \\
\hline $24 / M / 72$ & OU & NA & NA \\
\hline $25 / F / 47$ & OU & OD & NA \\
\hline $26 / F / 55$ & OU & NA & Diabetes mellitus \\
\hline
\end{tabular}

Abbreviations: NA, not applicable; SRN, subretinal neovascularization.
1A) was defined as focal exudative telangiectasia limited to 2 clock hours or less in the juxtafoveal areas in 8 of the patients. In our experience, cases with 2 clock hours progress to more extensive disease with time.

In group 2 (equivalent to perifoveal telangiectasia in our series), a subcategory was based on the age at onset. Ninety-two patients were classified as group 2A, and 2 young brothers aged 9 and 12 years were placed in group 2B. These 2 brothers represent rare cases, possibly a familial disorder not characteristic of group 2 as a whole. Only 1 other article ${ }^{12}$ exists in the ophthalmic literature on perifoveal telangiectasia in young individuals. That case study, however, involved familial telangiectasia and spastic paraplegia, not exactly an idiopathic presentation. Furthermore, group 2A was also subdivided into 5 stages of the disease as follows: stage 1, or occult vascular abnormalities evident only by mild staining with fluorescein; stage 2, or mild loss of transparency without clinically evident telangiectatic vessels; stage 3, or prominent dilated right-angle retinal venules; stage 4, or retinal pigment hyperplasia extending into the retina; and stage 5, or SRN originating from proliferation of intraretinal capillaries beneath the neurosensory retina. A method for staging a disease is usually based on the time sequence of progressive changes that occur as part of the natural course of the disorder. Most of the findings in group 2 occur in some patients but not others at various stages of the disease. We prefer to classify this group into 2 stages, 1 with nonproliferative vascular abnormalities and 1 with proliferative vascular abnormalities.

Group 3 (or occlusive telangiectasia in our series) was rarely seen and was subdivided by Gass and Blodi ${ }^{1}$ into 2 subgroups. Three women with related systemic vascular occlusive or inflammatory disease constituted group 3A, and 4 men with central nervous system involvement composed group B. In the Gass-Blodi series, there was a total of 140 cases seen over a 28-year period: 39 cases in group 1,94 in group 2, and 7 in group 3. Their case frequency ratio in the series was 1.0:3.1:0.2 cases, respectively.

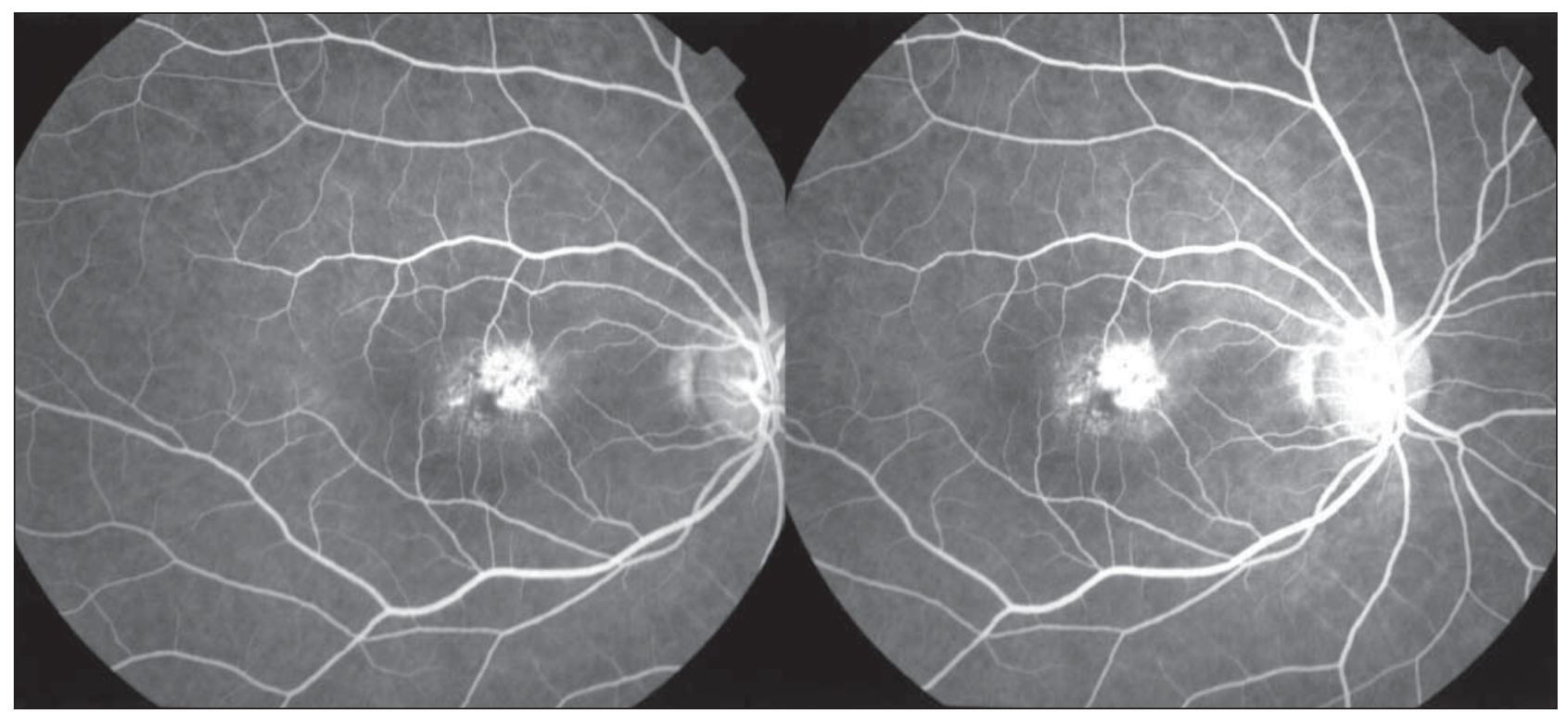

Figure 7. Perifoveal telangiectasia in the nonproliferative stage. The stereo photograph shows superficial nasal and deep perifoveolar retinal leakage cascading in the central macula. 

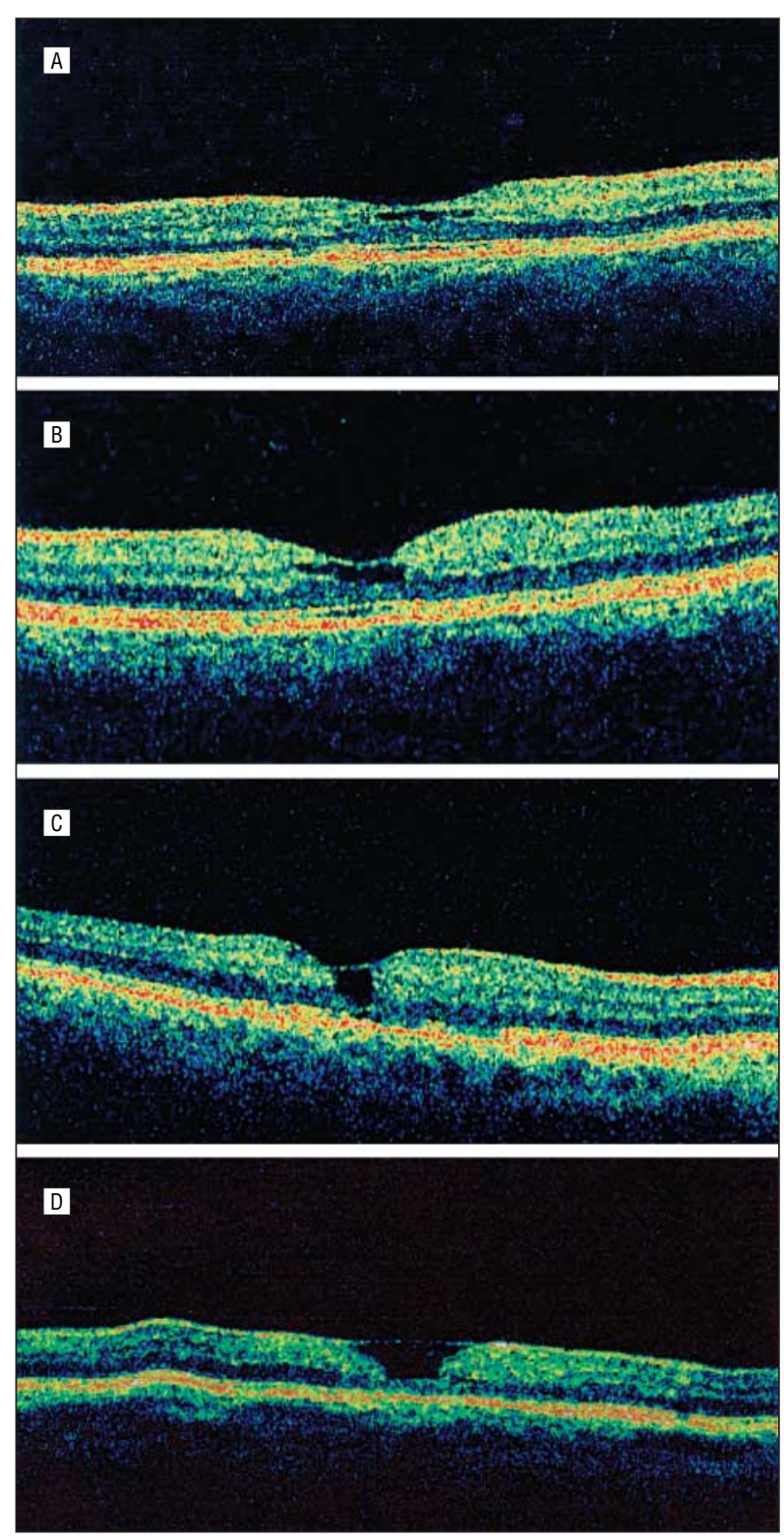

Figure 8. Perifoveal telangiectasia in the nonproliferative stage. The optical coherence tomographic scans show progression of inner lamellar cystic spaces and foveal atrophy that correlates with visual acuity loss. The visual acuities are 20/30 (A), 20/40 (B), 20/70 (C), and 20/100 (D). Note that there is no cystoid change evident in the perifoveal area.

Overall, the results of our study are quite similar to the Gass-Blodi series. ${ }^{1}$ First, the case frequency ratio was almost identical, approximately 1 type 1 case to 3 type 2 cases in each series. Our study also confirmed the rarity of type 3, the occlusive variant; 7 cases were seen by Gass ${ }^{1}$ in 28 years, and none were noted in our study in 3 years. However, some dissimilarity exists between the series by Gass and Blodi and our series, and there are new observations, both clinical and imaging, in our study. Based on our findings, we propose a simplified revision of the Gass-Blodi classifications.

\section{TYPE 1: ANEURYSMAL TELANGIECTASIA}

Groups 1A and 1B were merged by omitting the distinctions based on 2 clock hours of involvement in the jux- tafoveal area. We believe that a small focal area in the juxtafoveal region is a less severe variant in the spectrum of the disease (Figure 6A and B). Most cases of aneurysmal telangiectasia were in men and were unilateral. An exception in our series was a woman with bilateral disease. Gass and Blodi ${ }^{1}$ did not mention the sex of their 2 bilateral cases. There was no related systemic disease in the Gass-Blodi series or in our series.

As in the article by Gass and Blodi, ${ }^{1}$ patients with aneurysmal telangiectasia varied considerably in their clinical features. As previously described, ${ }^{13}$ the intraretinal microangiopathy changes in our series were evident in the superficial and deep circulations with leakage into the retina and cystoid macular edema. We found minimal ischemia and no evidence of neovascularization (neither preretinal neovascularization nor SRN). Lipid deposition was a characteristic feature, but there was no pigment proliferation (Figure 5). There were also no vitreoretinal crystalline deposits or signs of disciform scarring. A key to the nature of this vasculopathy was its distribution in the fundus. Although a vascular lesion in the paramacular or juxtafoveal area was essential in its diagnosis, this group may also develop focal vascular change in the midperipheral fundus and even in the more anterior fundus (Figure 5). This condition may be considered within the spectrum of Coats' disease (both this series and the Gass-Blodi series ${ }^{1}$ excluded patients with macular and widespread or diffuse telangiectasia). We consider aneurysmal telangiectasia to be a form of Coats' disease that is found in the macula. Our study was able to incorporate OCT findings in evaluating these patients. The OCT images in patients with aneurysmal telangiectasia clearly documented and confirmed the presence and degree of macular edema and foveal exudative cystic changes, which were present in all of the cases. The edema was usually evident clinically and angiographically. In some eyes, however, the edema extended beneath the retina to produce shallow detachments of the macula that were imaged only with OCT (Figure 6F). Although larger aneurysms produced shadowing of the posterior retina, these structures were also visible clinically and angiographically; where the subretinal fluid was associated with turbid exudation, there was corresponding photoreflectance and posterior shadowing (Figure 6F). The known histopathological findings for this group are consistent with our clinical observations of dilation of capillaries, aneurysms, leakage, and minimal nonperfusion. ${ }^{14}$

\section{TYPE 2: PERIFOVEAL TELANGIECTASIA}

As in the Gass-Blodi classification, ${ }^{1}$ the patients with perifoveal telangiectasia in our series had clinical findings limited to the perifoveolar area. The demographics were also similar. There was no sex difference, and all of the cases were bilateral. In our series, the mean age at onset was 59 years, which was similar to the mean age of 55 years in the Gass-Blodi series. Five patients (19.2\%) in this group had diabetes mellitus. In a mixed racial population of patients with an average age of 59 years, this is not likely to be a significant association. The Gass-Blodi series did not include patients with diabetes mellitus at the time of diagnosis. Chew et $\mathrm{al}^{15}$ did find diabetes mellitus to 

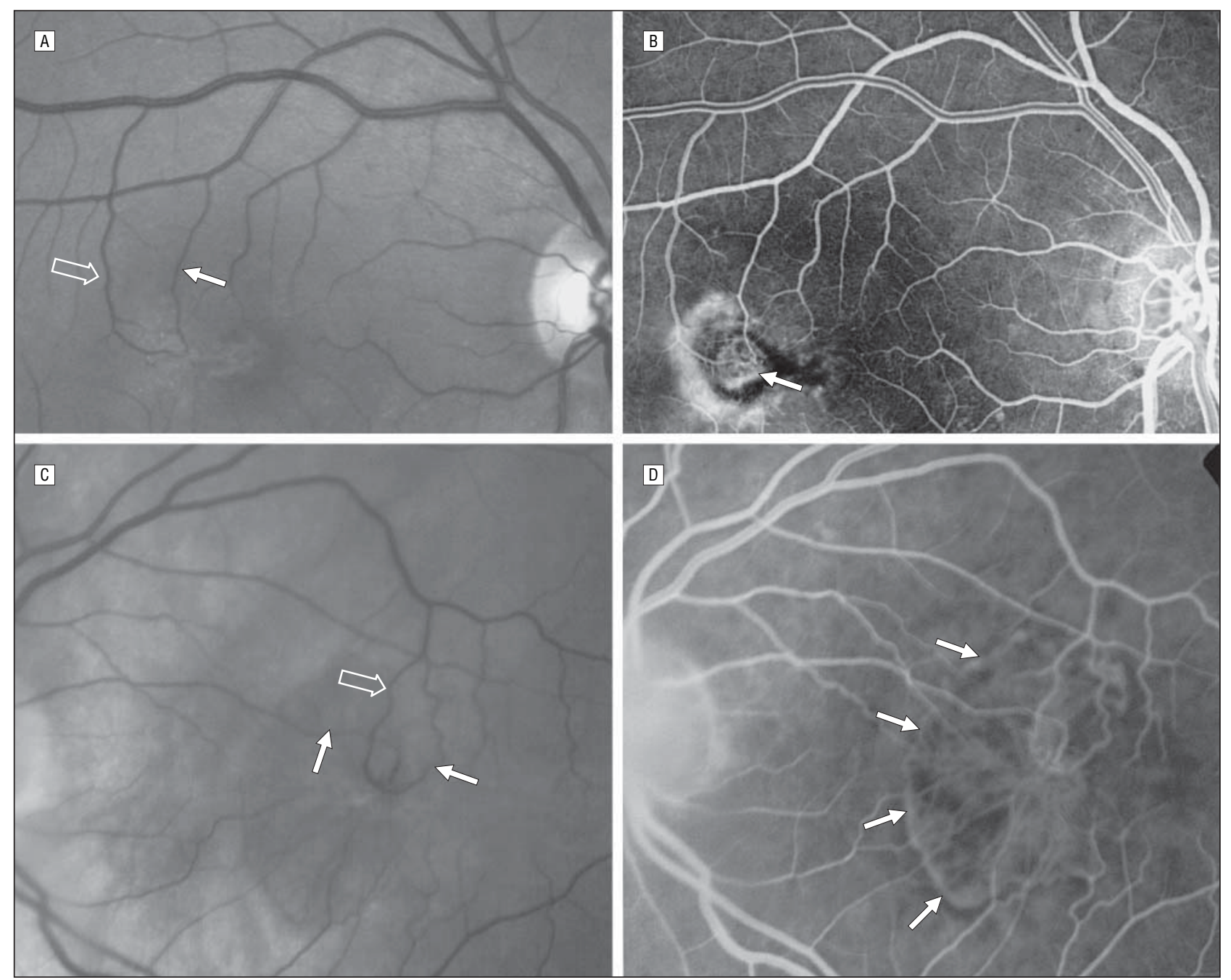

Figure 9. Perifoveal telangiectasia in the proliferative stage. A, There is subretinal neovascularization with a retinal-retinal anastomosis. The perfusing arteriole (solid arrow) and the draining venule (open arrow) are in the retina. B, The retinal-retinal anastomosis appears to overlie the subretinal neovascularization (arrow). $\mathrm{C}$, There is a retinal-retinal anastomosis with 2 perfusing arterioles (solid arrows) and a draining venule (open arrow). D, The retinal-retinal anastomosis communicates with nonpigmented subretinal neovascularization (arrows).

be a risk factor. In our series, we removed the GassBlodi group 2B subcategory that was based on age at onset. Group 2B comprised only 2 brothers who were aged 9 and 12 years. This familial variant is extremely rare and, to our knowledge, has not been described in any other series.

Loss of retinal transparency, small telangiectatic vessels, fluorescein leakage, and a cystic appearance to the fovea without intraretinal leakage were the predominant and most consistent clinical features in our series (Figure 2). The loss of retinal transparency typically appeared grayish.

Cascading retinal vascular abnormalities, demonstrated with stereoangiography but not previously described, were evident in some of our cases (Figure 7). Curiously, the deep retinal circulation that was clearly demonstrated with fluorescein angiography was not seen in other retinal microvasculopathies in the macula. The dilated right-angle vessels described by Gass and Blodi ${ }^{1}$ proved in our series to be both venular and arteriolar in nature (Figure 9). Furthermore, we observed intraretinal and subretinal anastomoses, which were not previ- ously described (Figure 9). These right-angle vessels may lead to a network of proliferating vessels in the deep retinal circulation. This network can extend beneath the retina to form a subretinal network, often with a clearly discernible RRA or retinal-subretinal anastomosis. The SRN was not necessarily associated with pigment epithelial hyperplastic plaques or retinal migration (Figure 9). ${ }^{1}$ Unlike choroidal neovascularization, which is usually associated with pigment epithelial proliferation, our series indicated that the SRN seldom had an envelope or plaque of pigment hyperplasia (Figure 3 and Figure 9). This was also noted in the article on excision of SRN in perifoveal telangiectasia by Berger et al. ${ }^{6} \mathrm{New}$ vessels rising from the choroid are not characteristic of type II, but 1 of our cases appears to be an exception, as a pigmented membrane communicating with the retinal vasculature evolved with a hemorrhage and fibrosis into a disciform scar (Figure 10). The retinal pigment epithelium (RPE) in these patients with perifoveal telangiectasia was healthy compared with that in patients with age-related macular degeneration. Whereas an RPE detachment is common in age-related macular degenera-

$$
\text { (REPRINTED) } \bar{A} \text { ARCH OPHTHALMOL/VOL 124, APR } 2006 \quad \text { WWW.ARCHOPHTHALMOL.COM }
$$




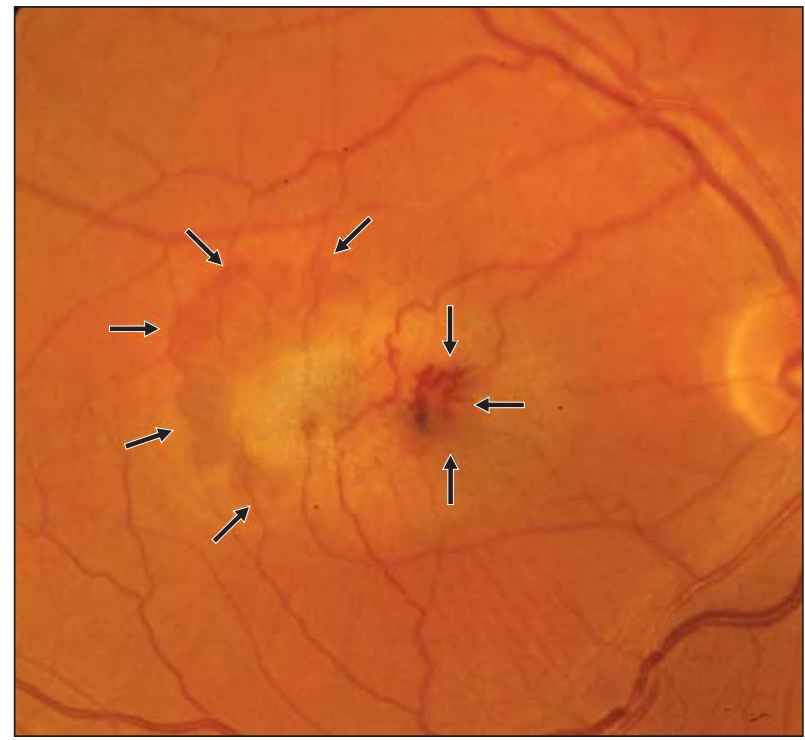

Figure 10. Perifoveal telangiectasia in the proliferative stage. There is subretinal hemorrhage (arrows) adjacent to a clinically pigmented membrane presumed to be due to choroidal neovascularization.

tion, it is rare in perifoveal telangiectasia. One of us (L.A.Y.) has seen an RPE detachment in perifoveal telangiectasia only twice over the past 35 years, perhaps as a coincidental finding in these patients in the sixth decade of life.

To our knowledge, the precise mechanism in perifoveal telangiectasia for the proliferation of retinal capillaries from the deep capillary circulation is unknown. In the absence of capillary ischemia or inflammation, progressive retinal endothelial cell degeneration may be the triggering, vasogenic cellular mechanism. Unlike diabetic microangiopathy, there is no preretinal proliferation. It is tempting to speculate that proliferation from the middle retinal circulation to extend beneath the retina is similar to experimental animal models or the neovascular form of age-related macular degeneration known as retinal angiomatous proliferation (RAP). ${ }^{16-18}$ These vasogenic correlates also do not have definite evidence of inflammation or ischemia as precursors. Patients with RAP are also known to develop RRAs. Furthermore, these RRAs appear to be compensatory in nature- to perfuse and to drain an expanding angiomatous process. Davidorf $\mathrm{et}^{\mathrm{al}}{ }^{7}$ noted a reduction in the caliber of a dilated perfusing arteriole and draining venule after surgical excision of the SRN in perifoveal telangiectasia. We have noticed similar vessel caliber changes following photodynamic therapy in perifoveal telangiectasia and age-related macular degeneration. The RRA within the retina in RAP and perifoveal telangiectasia eventually extends beneath the retina when SRN develops. However, unlike RAP, a retinal-choroidal anastomosis does not usually evolve since choroidal neovascularization is not typically involved in the vasogenic process of patients with perifoveal telangiectasia. ${ }^{19,20}$ We believe that the subretinal plaque of pigmentation in perifoveal telangiectasia is reactive hyperplasia induced by extension of the retinal vascular proliferation posteriorly to stimulate RPE cells, again similar to RAP. Lafaut et al ${ }^{21}$ described this occurring in histopathological specimens of RAP. We hypothesize that this mechanism for the pig- mentary response is more likely than the opposite (RPE cell migration into the retina to stimulate vascular proliferation) proposed by Gass and Owakawa. ${ }^{11}$

Our clinical observations of IMT correlate well with previously described histopathological findings. In 1980, Green et $\mathrm{al}^{8}$ described thickening of retinal capillaries and narrowing of the caliber of the lumen on electron microscopical analysis. The thickening from marked proliferation of the basement membrane in a multilayered configuration was noted. Degeneration of pericytes, and occasionally endothelial cells, was also described. Surprisingly, these changes were also noted to a lesser degree in the peripheral fundus as well, where there are typically no clinically detectable abnormalities. Green and colleagues also noticed retinal capillary proliferation into the outer retina as far as the photoreceptor layer. Since this landmark article appeared, other clinical pathological correlations have added to our knowledge of IMT abnormalities. Choroidal neovascularization with retinalchoroidal anastomosis has been documented histopathologically in 1 case. ${ }^{11}$ Retinal-choroidal anastomosis is not typical in perifoveal telangiectasia, but it is commonly known that any event that disturbs the RPE may predispose to choroidal neovascularization, particularly in elderly patients.

The OCT changes in perifoveal telangiectasia that were described in our series but not in the article by Gass and Blodi ${ }^{1}$ are revealing. The first detectable change is retinal thickening, not evident clinically but imaged with OCT (Figure 2). This finding could constitute a noninvasive way of making a diagnosis in cases where the telangiectatic vessels are occult in nature. On OCT imaging, cystic change at the fovea is an inner lamellar cyst bordered anteriorly by the inner limiting membrane (Figure 2). Lee et $\mathrm{al}^{22}$ also observed this finding in a singular case report of perifoveal telangiectasia. We believe that the term cyst rather than pseudohole is the most appropriate description of this abnormality since it is highly likely that the inner and outer tissues are derived from the neuroepithelium. This change was previously described as simulating a lamellar retinal hole by Gass and Owakawa, ${ }^{11}$ who did not have the advantage of OCT imaging. The course of the visual status can be followed by documenting the progressive loss of the outer retina with OCT, again noninvasively. The progression of the cyst is possibly the result of Müller cell degeneration and cystic macular degeneration at the fovea. Some OCT imaging of clinical features is not of practical value. The crystalline deposits, for example, were too small to induce photoreflectance, and the pigment figures simply created nonspecific subretinal reflectance and posterior shadowing (Figure 3B). These lesions are too small to be imaged with the resolution of the system. Furthermore, OCT registration is also limited with regard to point-to-point or pixel-to-pixel correlation as well. However, Trabucchi et $\mathrm{al}^{23}$ reported nonspecific shadowing in a single case of perifoveal telangiectasia from RPE proliferation.

We prefer to classify perifoveal telangiectasia in 2 stages: the nonproliferative stage when there are exudative telangiectasia and foveal atrophy, and the proliferative stage with the advent of SRN. In the nonproliferative stage, the mechanism for vision loss is the exudative 


\begin{tabular}{|c|c|c|}
\hline \multirow[b]{2}{*}{ IJRT* } & \multicolumn{2}{|r|}{ IMT† } \\
\hline & Classification & Description \\
\hline Group 1 & Type 1 & Aneurysmal telangiectasia \\
\hline $1 \mathrm{~A}:$ visible and exudative IJRT & NA & NA \\
\hline 1B: visible, exudative, and focal IJRT & NA & NA \\
\hline Group 2 & Type 2 & Perifoveal telangiectasia (nonproliferative or proliferative) \\
\hline 2A: occult and nonexudative IJRT & NA & NA \\
\hline Stage 1: occult telangiectatic vessels & NA & NA \\
\hline $\begin{array}{l}\text { Stage 2: loss of transparency without clinically evident } \\
\text { telangiectatic vessels }\end{array}$ & NA & NA \\
\hline Stage 3: prominent dilated right-angle retinal venules & NA & NA \\
\hline Stage 4: retinal pigment hyperplasia into the retina & NA & NA \\
\hline Stage 5: SRN from proliferation of intraretinal capillaries & NA & NA \\
\hline 2B: juvenile occult familial IJRT & NA & NA \\
\hline Group 3 & NA & NA \\
\hline 3A: occlusive IJRT & NA & NA \\
\hline 3B: occlusive IJRT with CNS vasculopathy & NA & NA \\
\hline
\end{tabular}

Abbreviations: CNS, central nervous system; IJRT, idiopathic juxtafoveolar retinal telangiectasis; IMT, idiopathic macular telangiectasis; NA, not applicable; SRN, subretinal neovascularization.

* Gass-Blodi classification. ${ }^{1}$

†Our proposed classification.

change to a degree but principally the foveal atrophy, which is best monitored with OCT (Figure 2). In the proliferative stage, OCT is less helpful, except to detect occult macular detachments (Figure 3 ). When subretinal new vessels have developed, cystoid macular edema, subretinal hemorrhage, and fibrovascular proliferation are usually evident clinically and angiographically. In the proliferative stage, the principle method for vision loss changes from foveal atrophy to fibrosis. Thus, this simple staging of perifoveal telangiectasia has an anatomical basis and a functional significance. The other typical characteristics of perifoveal telangiectasia, such as pigment plaques and crystalline deposits, occur variably in the course of the disease and may persist without visual consequence for years.

\section{TYPE 3: OCCLUSIVE TELANGIECTASIA}

Occlusive telangiectasia has been described previously by Gass and Blodi. ${ }^{1}$ In their revised classification, 7 of 140 cases were described in this group. Three cases had systemic vascular occlusive disease and 4 had familial ocular cerebral occlusive disorders. Furthermore, occlusive telangiectasia has very little to do with telangiectasia. It is an ischemic foveal disease bordered by a few dilated capillaries that are most likely compensatory changes responding to the capillary nonperfusion. Accordingly, we suggest that this type be omitted from the macular idiopathic telangiectasia classification. In our opinion, it is best viewed as a perifoveal capillary nonperfusion, as an ocular manifestation of systemic or cerebral familial disease.

In summary, we proposed a new classification of IMT. The previous and proposed classifications are in Table 3. Type 1 (aneurysmal telangiectasia) is a variable but generally unilateral disease seen mostly in men and is an exudative telangiectasia associated with microaneurysms and macroaneurysms that leak into the macula and sometimes beyond. There is no pigment, crystalline deposit, or neovascularization in this type. The leakage is associated with cystic change in the macula, clearly documented with OCT. Type 2 (perifoveal telangiectasia) is a bilateral perifoveolar telangiectatic disease with no sex predisposition. It is limited to the perifoveal area and is not associated with aneurysms or cystoid leakage. In the nonproliferative stage, there is an exudative telangiectasis and foveal atrophy. In the proliferative stage, there is SRN and fibrosis. This simple classification will lead to a better understanding of the entities by ophthalmologists and retinal specialists. It will also assist in teaching, in future research, and hopefully in developing new methods of therapy.

Submitted for Publication: February 14, 2005; final revision received May 24, 2005; accepted June 10, 2005. Correspondence: Lawrence A. Yannuzzi, MD, LuEsther T. Mertz Retinal Research Center, Manhattan Eye, Ear, and Throat Hospital, 210 E 64th St, New York, NY 10021 (vrmny@aol.com).

Financial Disclosure: None.

Funding/Support: This study was supported by the Macula Foundation, Inc, New York, NY.

Acknowledgment: We wish to thank the retinal specialists of Vitreous-Retina-Macula Consultants of New York, New York (Yale Fisher, MD, Jay Klancnik, MD, John Sorenson, MD, and Richard Spaide, MD) for their assistance in recruiting patients for this series.

\section{REFERENCES}

1. Gass JD, Blodi BA. Idiopathic juxtafoveolar retinal telangiectasis: update of classification and follow-up study. Ophthalmology. 1993;100:1536-1546.

2. Axer-Siegel R, Bourla D, Priel E, Yassur Y, Weinberger D. Angiographic and flow patterns of retinal choroidal anastomoses in age-related macular degen- 
eration with occult choroidal neovascularization. Ophthalmology. 2002;109: 1726-1736.

3. Puliafito CA, Hee MR, Lin CP, et al. Imaging of macular diseases with optical coherence tomography. Ophthalmology. 1995;102:217-229.

4. Hee MR, Izatt JA, Swanson EA, et al. Optical coherence tomography of the human retina. Arch Ophthalmol. 1995;113:325-332.

5. Voo I, Mavrofrides EC, Puliafito CA. Clinical applications of optical coherence tomography for the diagnosis and management of macular diseases. Ophthalmol Clin North Am. 2004;17:21-31.

6. Berger AS, McCuen BW II, Brown GC, Brownlow RL Jr. Surgical removal of subfoveal neovascularization in idiopathic juxtafoveolar retinal telangiectasis. Retina. 1997:17:94-98.

7. Davidorf FH, Pressman MD, Chambers RB. Juxtafoveal telangiectasis: a name change? Retina. 2004;24:474-478.

8. Green WR, Quigley HA, De la Cruz Z, Cohen B. Parafoveal retinal telangiectasis: light and electron microscopy study. Trans Ophthalmol Soc U K. 1980;100:162-170.

9. Eliassi-Rad B, Green WR. Histopathologic study of presumed parafoveal telangiectasis. Retina. 1999;19:332-335.

10. Centers for Disease Control and Prevention. National Diabetes Fact Sheet: General Information and National Estimates on Diabetes in the United States, 2002. Atlanta, Ga: Centers for Disease Control and Prevention, US Dept of Health and Human Services; 2003.

11. Gass JD, Owakawa RT. Idiopathic juxtafoveolar retinal telangiectasis. Arch Ophthalmol. 1982;100:769-780.

12. Leys A, Gilbert HD, Van De Sompel W, et al. Familial spastic paraplegia and maculopathy with juxtafoveolar retinal telangiectasis and subretinal neovascularization. Retina. 2000;20:184-189.
13. Lee ST, Friedman SM, Rubin ML. Cystoid macular edema secondary to juxtafoveolar telangiectasis in Coats' disease. Ophthalmic Surg. 1991;22:218-221.

14. Tripathi R, Ashton N. Electron microscopical study of Coats' disease. Br J Ophthalmol. 1971;55:289-301.

15. Chew EY, Murphy RP, Newsome DA, Fine SL. Parafoveal telangiectasis and diabetic retinopathy. Arch Ophthalmol. 1986;104:71-75.

16. Okamoto N, Tobe T, Hackett SF, et al. Transgenic mice with increased expression of vascular endothelial growth factor in the retina: a new model of intraretinal and subretinal neovascularization. Am J Pathol. 1997;151:281-291.

17. Heckenlively JR, Hawes NL, Friedlander M, et al. Mouse model of subretinal neovascularization with choroidal anastomosis. Retina. 2003;23:518-522.

18. Yannuzzi LA, Negrao S, lida $T$, et al. Retinal angiomatous proliferation in agerelated macular degeneration. Retina. 2001;21:416-434.

19. Engelbrecht NE, Aaberg TM Jr, Sung J, Lewis ML. Neovascular membranes associated with idiopathic juxtafoveolar telangiectasis. Arch Ophthalmol. 2002; 120:320-324.

20. Gass JD. Chorioretinal anastomosis probably occurs infrequently in type $2 A$ idiopathic juxtafoveolar retinal telangiectasis. Arch Ophthalmol. 2003;121:13451346.

21. Lafaut BA, Aisenbrey S, Vanden Broecke C, Bartz-Schmidt KU. Clinic pathological correlation of deep retinal vascular anomalous complex in age related macular degeneration. Br J Ophthalmol. 2000;84:1269-1274.

22. Lee HC, Liu M, Ho AC. Idiopathic juxtafoveal telangiectasis in association with celiac sprue. Arch Ophthalmol. 2004;122:411-413.

23. Trabucchi G, Brancato R, Pierro L, Introini U, Sannace C. Idiopathic juxtafoveolar retinal telangiectasis and pigment epithelial hyperplasia: an optical coherence tomographic study. Arch Ophthalmol. 1999;117:405-406.

\section{Call for Papers: Ocular Genetics Theme Issue}

The ARCHIVES will be publishing a theme issue on ocular genetics to provide an overview of the status of this rapidly changing field. The issue will (1) include reviews of the genetics of major eye diseases, (2) provide insight into the current approaches and tools needed to evaluate findings from genetic studies (eg, statistical genetics and genetic epidemiology), (3) suggest how these tools will be used in the future to advance understanding of the etiology, pathogenesis, and treatment of different eye conditions, and (4) provide clinicians with some guidelines for communicating genetic risks to the average patient. The issue will include a combination of invited papers and papers presenting original research. Leslie Hyman, PhD, Janey Wiggs, MD, PhD, Barbara Klein, MD, MPH and Barbara Nemesure, $\mathrm{PhD}$ will be guest editors for this special issue. We invite and encourage all investigators to submit manuscripts describing original ocular genetics research by July 1, 2006. All manuscripts submitted for this issue are subject to an expedited peer review. Early receipt ensures the best chance for acceptance for this special issue. Accepted manuscripts not included in this issue will be published in other issues of the ARCHIVES. Please note in the cover letter that the submission is for the ocular genetics theme issue. The expected publication date for this issue is January 2007. 